\title{
Time-Resolved Emission from Semiconductor Microcavities
}

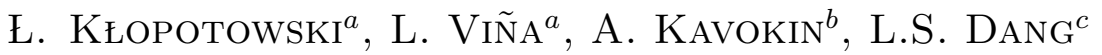 \\ AND R. ANDRÉ ${ }^{c}$ \\ ${ }^{a}$ Departamento de Física de Materiales, Universidad Autónoma de Madrid \\ Cantoblanco, 28049 Madrid, Spain \\ ${ }^{b}$ LASMEA, UMR 6602 CNRS, Université Blaise-Pascal \\ 24, av des Landais, 63177 Aubiére, France \\ ${ }^{c}$ Laboratoire de Spectrométrie Physique, Université Joseph Fourier \\ 38402 Grenoble, France
}

\begin{abstract}
We present a time-resolved study of the light emission of a CdTe-based microcavity. In the nonlinear regime, under high excitation conditions, in the strong coupling regime, we observe pronounced beats of the intensity of the photoluminescence arising from the bottleneck region of the excitonpolariton band. These beats are very sensitive to the excitation density and vanish under weak pumping conditions. We attribute the beats to a new nonlinear coupling mechanism of optically active and dark crystal states, related to polariton-polariton scattering, which leads to mixing between bright and dark states.
\end{abstract}

PACS numbers: 71.36.+c, 71.55.Gs, 78.47.+p

\section{Introduction}

Semiconductor microcavities allow us to precisely control the radiation-matter interaction. This interaction is the strongest when the characteristic frequencies of photons (radiation) and excitons (matter) are brought into resonance. Two different regimes can be established under this resonance condition: the strong and weak coupling regimes. The largest effort has been devoted to the study of the strong coupling regime (SCR), in which the eigenstates of the system are no longer pure exciton or photon but a superposition of both, known as cavity-polaritons [1]. The resonant frequencies of excitons and photons are split, leading to the so-called Rabi splitting, in analogy with atomic cavities [2]. On the contrary, in the weak 
coupling regime (WCR), the polaritons are bleached. A transition from the SCR to the WCR can occur, for example, when the quantum wells (QWs) are close to a node of the standing wave in the cavity or when the exciton population is very large [3]. The latter situation hindered the study of the nonlinear properties of cavity polaritons. Only in the last years it has been possible to observe the polariton nonlinear emission in both III-V [4-6] and II-VI [7-10] semiconductor microcavities. In particular, in the work of Dang et al. [7], a transition to the nonlinear regime for moderate excitation densities is observed, and a transition to the WCR is found only for much larger powers.

Another issue that has drawn a lot of attention in the nonlinear SCR is the existence of a polariton-polariton scattering mechanism stimulated by the final state population. This mechanism will be active in a bosonic system, such as cavity polaritons, as soon as the final state population approaches the unity. Clear experimental evidence of this stimulated scattering has been reported recently in the literature $[5,11]$. In those experiments, the parametric scattering was enhanced by a convenient choice of the angle of incidence of the excitation beams. The result is a macroscopic polariton occupancy ("condensation") of the states at $\boldsymbol{K} \sim 0$ and $\boldsymbol{K} \sim 2 \boldsymbol{k}_{\text {pump }}$, where $\boldsymbol{k}_{\text {pump }}$ is the incident pump wave vector.

Here we present experimental evidence of a new nonlinear effect linked to polariton-polariton interactions. We have observed quantum beats between optically active polariton states and spin-forbidden exciton states coupled by a polariton-polariton (exciton-exciton) scattering process. This observation is a manifestation of a new kind of parametric oscillation process in microcavities that does not require resonant pumping and keeps coherence of the polariton (exciton) system much longer than the polariton radiative lifetime (up to $100 \mathrm{ps}$ in our experiment). It shows the remarkable potential of microcavities for realization of quantum optical devices.

In most quantum wells (QWs), the energy of a heavy-hole is typically lower than that of a light-hole hence the exciton ground state is formed by an electron ( $\operatorname{spin} S= \pm 1 / 2$ ) and a heavy-hole (spin $J= \pm 3 / 2$ ). The entire exciton spin thus has $S+J= \pm 1$ and $S+J= \pm 2$ projections on the structure axis allowed for the ground state. The states with spin projection \pm 1 are optically active (bright), as they can be excited by a right- or left-circular polarized light beam. They are coupled with a photonic mode of the cavity and participate in formation of the exciton-polaritons. On the other hand, the optical excitation of \pm 2 states is forbidden by the selection rules. These are so-called dark states, completely decoupled from the photonic modes. Dark and bright exciton states are typically split by a few tens of $\mu \mathrm{eV}$. Mixed coherent excitation of these states may result in oscillations of their populations, i.e. quantum beats. The mixing can be achieved by application of an in-plane magnetic field as has been experimentally demonstrated in QWs [12] and recently in microcavities [13]. This is a linear optical effect which does not involve any parametric oscillation. 


\section{Experimental details}

Our sample is a $\mathrm{Cd}_{0.40} \mathrm{Mg}_{0.60} \mathrm{Te}$ microcavity of thickness $\lambda$, sandwiched between top (bottom) distributed Bragg reflectors (DBRs). These mirrors are made of 17.5 (23) pairs, of alternating $\lambda / 4$ thick layers of $\mathrm{Cd}_{0.40} \mathrm{Mg}_{0.60} \mathrm{Te} / \mathrm{Cd}_{0.75} \mathrm{Mn}_{0.25}$ Te. Two pairs of $90 \AA$ thick CdTe quantum wells are placed at the antinodes of the cavity, leading to a Rabi splitting of $\sim 14 \mathrm{meV}$. The optical excitation was performed at the first minimum above the stop-band of the DBRs, $\sim 80 \mathrm{meV}$ higher than the emission energy of the cavity-like polariton branch, to assure the same excitation conditions in all the experiments. A slight wedge of the cavity thickness allows the tuning of the cavity and the exciton into resonance by moving the excitation spot across the wafer [14].

The experiments were performed in a variable temperature cold-finger cryostat. The samples were optically excited with light pulses from a Ti:sapphire mode-locked laser pumped by an $\mathrm{Ar}^{+}$-ion laser. The photoluminescence (PL), angle-resolved using a small pinhole which gives a resolution of $1^{\circ}$, was detected at the lower polariton $(\mathrm{LP})$ branch $(1.61 \mathrm{eV}$ at $\boldsymbol{K}=0)$ and time- and spectrally-resolved PL was detected using a streak camera with an overall time resolution of $\sim 6$ ps. For polarization-resolved measurements, a pair of $\lambda / 4$ plates are included in the experiment: the excitation light is $\sigma^{+}$-polarized and the $\mathrm{PL}$ emission is analyzed into its $\sigma^{+}$- and $\sigma^{-}$-polarized components. The experiments are performed at negative detunings with power densities below $50 \mathrm{~W} \mathrm{~cm}^{-2}$, ensuring the persistence of the strong-coupling regime.

\section{Results and discussion}

Figure 1 depicts, on a semi-logarithmic scale, the time evolution of the emission at $\boldsymbol{K}=0$, exciting with $\sigma^{+}$-polarized pulses and detecting the co-polarized emission $\left(\sigma^{+}\right)$, for a detuning of $\delta=-13 \mathrm{meV}$, at different power densities ranging from 0.5 to $40 \mathrm{~W} \mathrm{~cm}^{-2}$. The rise-time is practically independent of the excitation power in the range used in these experiments, although a closer look at the initial curvature of the time-traces reveals that this curvature changes from negative (low excitation) to positive (high excitation), as has been described previously [15]. A positive curvature indicates a relaxation mechanism that is based on an increasing rate, like final state stimulation [16]. The decay time decreases from $\sim 300 \mathrm{ps}$ (at $0.5 \mathrm{~W} \mathrm{~cm}^{-2}$ ) to $150 \mathrm{ps}\left(\right.$ at $20 \mathrm{~W} \mathrm{~cm}^{-2}$ ) - solid lines are the corresponding fits - and at higher powers an additional initial much faster decay is found with a decay constant of $\sim 10 \mathrm{ps}$, while the slower decay remains constant at $150 \mathrm{ps}$ within experimental error.

Figure 2 shows, in a linear scale, the time-evolution of the photoluminescence, excited at the first minimum above the stop-band $(1.686 \mathrm{eV})$ and detected at the lower polariton branch $(1.61 \mathrm{eV})$, for an angle of detection of $9.4^{\circ}$ for the same detuning as in Fig. 1. At this high pumping intensity, $40 \mathrm{~W} \mathrm{~cm}^{-2}$, the PL intensity 


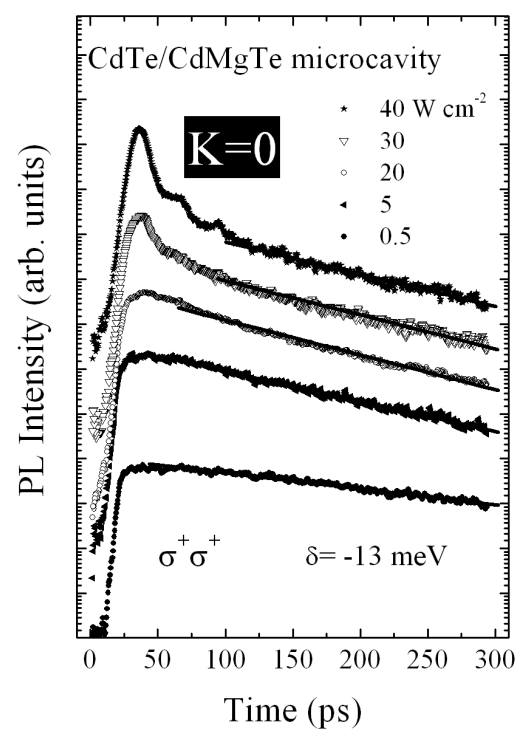

Fig. 1. Time evolution, on a semi-logarithmic scale, of the PL from the LP branch of the $\mathrm{CdTe} / \mathrm{CdMgTe}$ microcavity at $\boldsymbol{K}=0$ at a detuning of $-13 \mathrm{meV}$, for different excitation densities. The excitation is $\sigma^{+}$-polarized and the emission is analyzed into its $\sigma^{+}$component.

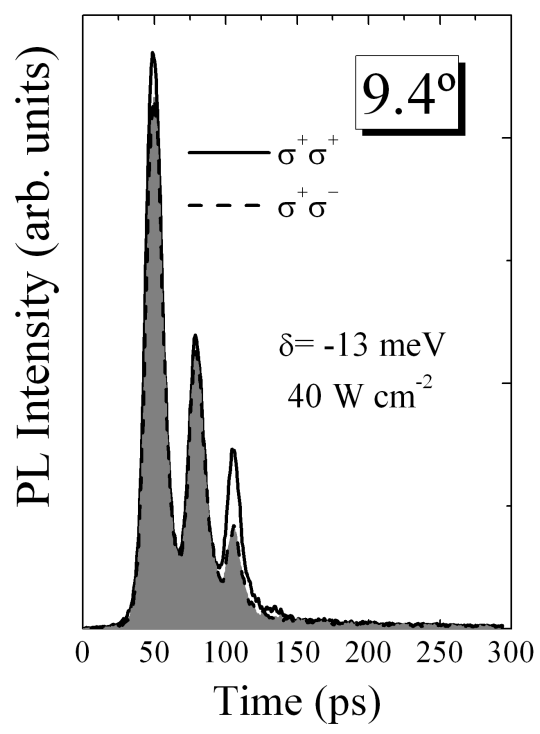

Fig. 2. Temporal evolution, on a linear scale, of the PL from the bottleneck region (at angle $9.4^{\circ}$ ) of our microcavity under nonresonant pumping at high-excitation. The solid and dashed lines correspond to $\sigma^{+}$- and $\sigma^{-}$-polarized emission, respectively. The pumping light is always $\sigma^{+}$-polarized. 
exhibits oscillations in both circular polarizations. Remarkably, these oscillations are in phase, so that they cannot be caused by any kind of beating between $\sigma^{+}$ and $\sigma^{-}$-polarized polariton states. The intensities of $\sigma^{+}$and $\sigma^{-}$components of the PL signal are rather similar, indicating that spin-relaxation of polaritons was quite efficient and that their \pm 1 spin states are nearly equally populated. However, the population of these two "bright" states oscillates with a period of about $30 \mathrm{ps}$. These oscillations survive over 100 ps which exceeds by two orders of magnitude the radiative lifetime of exciton-polaritons in the photon-like part of their lower dispersion branch.

These oscillations closely recall the beats between bright- and dark-exciton states observed by Renucci et al. [13]. But in contrast with the Renucci experiment, no magnetic field was applied to the sample in our case. Also, the effect we have observed is strongly nonlinear: at low pumping intensities we do not observe any beats; they only appear at strong enough pumping.

These experimental facts lead us to conclude that in the nonlinear regime some new mixing mechanism appears that allows the dark states to be populated and leads to quantum beats between dark excitons and exciton-polaritons. This mechanism must conserve the spin and optical coherence in the system; otherwise quantum interference between different states would be impossible. We believe that this mechanism is exchange interaction between polaritons or dark excitons with opposite spins. Let us assume that a pair of polaritons with spins +1 $(S=-1 / 2, J=3 / 2)$ and $-1(S=1 / 2, J=-3 / 2)$ exchange their electrons. The resulting states will have spins $+2(S=1 / 2, J=3 / 2)$ and $-2(S=-1 / 2$, $J=-3 / 2$ ), respectively. Thus a pair of dark excitons appears. A new exchange of electrons converts it into a pair of bright polaritons again, etc. A hole exchange would yield the same result, but as it is much less probable [17], we shall neglect it in the following discussion.

The energies of polaritons and dark excitons are split. It is clear that the process described here would be efficient only if the matrix element of polaritonpolariton (exciton-exciton) scattering, $W$, is comparable with this splitting. Following Tassone et al. [18], we estimate $W \sim E_{\mathrm{B}} a_{\mathrm{B}}^{2} / S$, where $E_{\mathrm{B}}$ is the exciton binding energy, $a_{\mathrm{B}}$ is an exciton radius, $S$ is the area of the system assumed to be given by the spot size of the incident laser, yielding $W \approx 10 \mu \mathrm{eV}$ in our case. Our experiment was performed under nonresonant pumping. The exciton-like part of the lower-polariton branch is populated first. Then, the polaritons relax toward the bottleneck region of the dispersion, where they can dwell a few tens of picoseconds before proceeding towards the photon-like part of the dispersion from where they escape radiatively. The beats between bright and dark states above the bottleneck lead to oscillations of the bright polariton population at the bottleneck and, consequently, in all lower states. That is why we observe oscillations of the PL intensity having the same phase for its $\sigma^{+}$and $\sigma^{-}$components. To describe the dynamics of such a process we introduce the following interaction Hamiltonian: 


$$
\begin{aligned}
H= & \sum_{k}\left(\varepsilon_{1} p_{1 \uparrow ; k}^{+} p_{1 \uparrow ; k}+\varepsilon_{1} p_{1 \downarrow ; k}^{+} p_{1 \downarrow ; k}+\varepsilon_{2} p_{2 \uparrow ; k}^{+} p_{2 \uparrow ; k}+\varepsilon_{2} p_{2 \downarrow ; k}^{+} p_{2 \downarrow ; k}\right) \\
& +\sum_{k, k^{\prime}} W_{k k^{\prime}}\left(p_{1 \uparrow ; k}^{+} p_{1 \downarrow ; k^{\prime}}^{+} p_{2 \uparrow ; k} p_{2 \downarrow ; k^{\prime}}+p_{1 \uparrow ; k} p_{1 \downarrow ; k^{\prime}} p_{2 \uparrow ; k}^{+} p_{2 \downarrow ; k^{\prime}}^{+}\right) .
\end{aligned}
$$

A complete and detailed resolution of this problem can be found in Ref. [19]. Figure 3 shows the calculated time evolution of the photoluminescence in the weakand strong-pumping regimes. The strong- and weak-pumping regimes correspond to different concentrations of polaritons $N_{0}$ injected into the system at $t=0$. We took $N_{0}=25 \times 10^{3}$ for the strong pumping limit and $N_{0}=5 \times 10^{3}$ for the weak pumping limit, respectively. One can see that in the first case pronounced

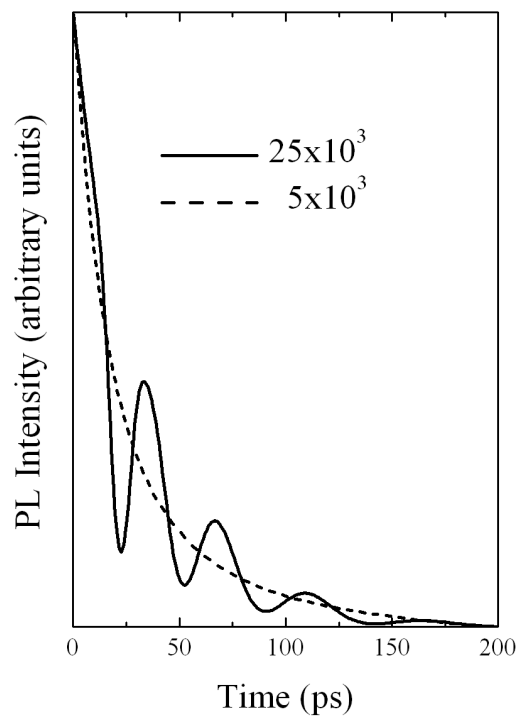

Fig. 3. Calculated intensity of the photoluminescence at angle $9.4^{\circ}$ from our microcavity in the strong pumping regime $\left(25 \times 10^{3}\right.$ injected polaritons, solid line $)$ and weak pumping regime $\left(5 \times 10^{3}\right.$ injected polaritons, dashed line $)$.

oscillations of the occupation numbers of bright states, and, consequently, of the photoluminescence intensity, appear. The period of these oscillations is very close to the experimentally observed $\sim 30 \mathrm{ps}$. On the other hand, with weaker pumping, no oscillations appear and a monotonic decay of the photoluminescence intensity is obtained, which is governed by the processes of radiative decay and slow scattering of the polaritons towards the ground state. These features are in very good agreement with our experimental observations despite the simplicity of the model used.

In order to demonstrate the robustness of the coherent interaction leading to the quantum beats between dark excitons and exciton-polaritons, we present in Fig. 4 the temperature dependence of the PL time-traces for a detuning of 


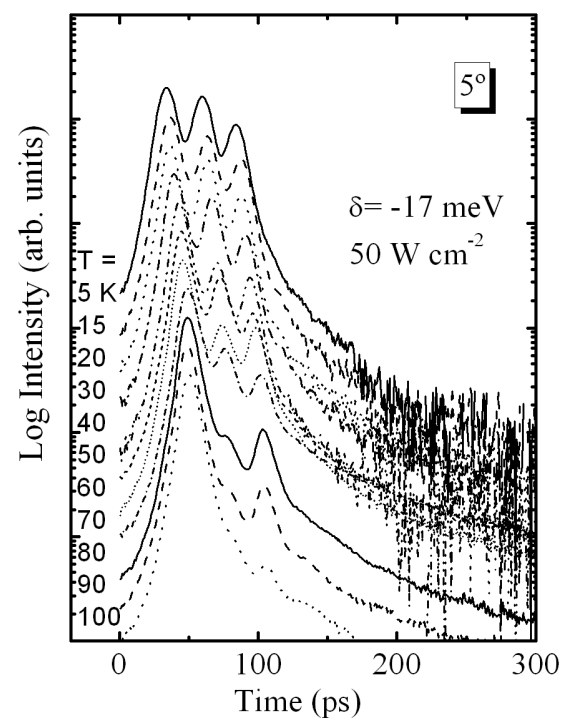

Fig. 4. Temporal evolution, on a semi-logarithmic scale, of the PL at angle $5^{\circ}$ from our microcavity at a detuning of $-17 \mathrm{meV}$, under nonresonant pumping at high-excitation, for different temperatures. The excitation is $\sigma^{+}$-polarized and the emission is analyzed into its $\sigma^{+}$component.

$-17 \mathrm{meV}$ and an emission angle of $5^{\circ}$ in the nonlinear regime. Different traces, which have been displaced vertically for clarity, for temperatures ranging from $5 \mathrm{~K}$ to $100 \mathrm{~K}$ are plotted in a semi-logarithmic scale. The beats are clearly observed up to the highest temperature shown in the figure, indicating that the coherence is maintained in spite of the increased phonon population.

\section{Conclusions}

In summary, we have reported strong oscillations in the time- , polarizationand angle-resolved light emission of a CdTe microcavity when the system is optically driven into the nonlinear regime. The period of the oscillations amounts to $30 \mathrm{ps}$ independently of the observation angle. We have also proposed a new mechanism of quantum oscillations in a semiconductor microcavity. The beats between exciton-polaritons and spin-forbidden ("dark") exciton states take place in a nonlinear regime due to polariton-polariton and exciton-exciton collisions. Remarkably, these collisions conserve the spin and coherence in the system that allows for observation of a kind of optical parametric oscillation, and survive at high temperatures. 


\section{Acknowledgments}

This work has been partially supported by the Spanish MCYT (MAT200200139), the CAM (07N/0042/2002) and the "Marie-Curie" MRTN-CT-2003503677 "Clermont2" ("Physics of Microcavities").

\section{References}

[1] C. Weisbuch, M. Nishioka, A. Ishikawa, Y. Arakawa, Phys. Rev. Lett. 69, 3314 (1992).

[2] S. Haroche, D. Kleppner, Physics Today 42, 24 (1989).

[3] R. Houdré, J.L. Gibernon, P. Pellandini, R.P. Stanley, U. Oesterle, C. Weisbuch, J. O'Gorman, B. Roycroft, M. Ilegems, Phys. Rev. B 52, 7810 (1995).

[4] P. Senellart, J. Bloch, Phys. Rev. Lett. 82, 1233 (1999).

[5] R.M. Stevenson, V.N. Astratov, M.S. Skolnick, D.M. Whittaker, M. Emam-Ismail, A.I. Tartakovskii, P.G. Savvidis, J.J Baumberg, J.S. Roberts, Phys. Rev. Lett. 85, 3680 (2000).

[6] G. Dasbach, T. Baars, M. Bayer, A. Larionov, A. Forchel, Phys. Rev. B 62, 13076 (2000).

[7] L.S. Dang, D. Heger, R. André, F. Boeuf, R. Romestain, Phys. Rev. Lett. 81, 3920 (1998).

[8] J. Bleuse, F. Kany, A.P. de Boer, P.C.M. Christianen, R. André, H. Umer-Tuffigo, J. Cryst. Growth 184-185, 750 (1998).

[9] F. Boeuf, R. André, R. Romestain, L.S. Dang, E. Péronne, J.F. Lampin, D. Hulin, A. Alexandrou, Phys. Rev. B 62, R2279 (2000).

[10] M. Mueller, J. Bleuse, R. André, Phys. Rev. B 62, 16886 (2000).

[11] P.G. Savvidis, J.J. Baumberg, R.M. Stevenson, M.S. Skolnick, D.M. Whittaker, J.S. Roberts, Phys. Rev. Lett. 84, 1547 (2000).

[12] I.V. Mashkov, C. Gourdon, P. Lavallard, D.Yu. Roditchev, Phys. Rev. B 55, 13761 (1997).

[13] P. Renucci, T. Amand, X. Marie, Physica E 17, 329 (2003).

[14] M.D. Martin, G. Aichmayr, L. Viña, R. André, Phys. Rev. Lett. 89, 077402 (2002).

[15] G. Aichmayr, M.D. Martin, L. Viña, R. André, unpublished.

[16] A. Vinattieri, J. Shah, T.C. Damen, D.S. Kim, L.N. Pfeiffer, M.Z. Maialle, L.J. Sham, Phys. Rev. B 50, 10868 (1994).

[17] M.Z. Maialle, E.A. de Andrada e Silva, L.J. Sham, Phys. Rev. B 47, 15776 (1993).

[18] F. Tassone, C. Piermarocchi, V. Savona, A. Quattropani, P. Schwendimann, Phys. Rev. B 56, 7554 (1997).

[19] I.A. Shelykh, L. Viña, A.V. Kavokin, N.G. Galkin, G. Malpuech, R. André, Phys. Status Solidi C 1, 1351 (2004). 